\title{
Teleoperación de un brazo robot Kinova MICO2 a través de un dispositivo Omni Bundle
}

\author{
Alex D. Paredes, Andrés Úbeda, Gabriel J. Garcia \\ Departamento de Física, Ingeniería de Sistemas y Teoría de la Señal \\ Carretera San Vicente del Raspeig, s/n, San Vicente del Raspeig, CP: 03690 \\ Universidad de Alicante \\ alex.paredes.a@gmail.com, \{andres.ubeda,gjgg\}@ua.es
}

\begin{abstract}
Resumen
En este trabajo se plantea efectuar el control remoto para entornos cotidianos de un brazo robótico Kinova MICO2 comandado por un dispositivo háptico Omni Bundle con realimentación de fuerza, dotando así de sensibilidad al operador. Se ha estudiado el método de control cinemático más efectivo de acuerdo a los requerimientos del sistema (en posición o en velocidad) $y$ se ha evaluado mediante la implementación de una interfaz de usuario para la monitorización y la configuración de los instrumentos utilizados. Como protocolo de comunicación, se ha establecido UDP, siendo la mejor opción en cuanto a velocidad y alcance. Los resultados muestran que un control PI (Proporcional-Integral) en velocidad es el más robusto y permite realizar tareas de teleoperación sin apenas error.
\end{abstract}

Palabras clave: teleoperación, dispositivo háptico, robot asistencial.

\section{INTRODUCCIÓN}

Como la mayoría de las innovaciones tecnológicas, la teleoperación surge de una necesidad, en este caso, la manipulación de objetos peligrosos en la industria nuclear, dando lugar al primer manipulador teleoperado mecánico, en 1948, denominado M1 [6]. Desde el planteamiento de este tipo de sistemas, se ha ido desarrollando más la tecnología, métodos y aplicaciones. Actualmente no es necesario que el dispositivo maestro y esclavo sean iguales, pudiendo tener diferente escala, o incluso tener modelos cinemáticos completamente diferentes, como es el caso del sistema propuesto en este estudio.

Un sistema de teleoperación común consta de un dispositivo maestro (interfaz háptica), que manipula la persona, y un dispositivo esclavo, que es el encargado de la manipulación de acuerdo a los comandos enviados por el maestro. El controlador acopla ambos dispositivos transmitiendo movimientos y fuerzas entre los mismos. Su objetivo es hacer que el control manual del operador sea robusto ante retardos, saturación de los actuadores y otras no linealidades e incluso ante errores propios del ser humano. También permite que los bucles de control tengan un comportamiento dinámico apropiado, reduciendo el trabajo del operador.

En este estudio se va a realizar un control teleoperado bilateral. En un control unilateral, el dispositivo maestro genera las señales de referencia, ya sea posición o velocidad, para el lazo de control del dispositivo esclavo, pero el operador no recibe las perturbaciones del entorno. Sin embargo, en el control bilateral, se agrega realimentación de fuerzas hacia el operador, cerrando el bucle de control. En este sentido, hay que tener en cuenta el retardo de teleoperación, provocado por varios factores, como la separación física entre el manipulador y el actuador, y el tipo de comunicación.

El campo de aplicación para esta tecnología es muy variado, desde la manipulación de materiales delicados con robots estáticos [3], hasta la exploración con robots móviles, como por ejemplo el robot Pioneer, o la evaluación del reactor nuclear de la planta de Chernobyl [1]. Se ha utilizado teleoperación también en robots de exploración espacial, como los de la NASA en expediciones a Marte [1]. En otros estudios se ha utilizado realidad aumentada, conjuntamente con dispositivos hápticos PHANToM para programar robots de soldadura [5]. Mucho más relacionado con esta investigación se ha utilizado este dispositivo háptico con realimentación de fuerza en un sistema para operación láser [8].

La propuesta de este estudio es utilizar el instrumento háptico Omni Bundle como dispositivo maestro para teleoperar un brazo robótico Kinova MICO2 en entornos cotidianos, con realimentación de esfuerzos, para dotar de sensibilidad del entorno al operador. Para ello se ha estudiado cuál es el tipo de control cinemático más adecuado y qué características debe tener el controlador para obtener los resultados más robustos. Por último, se han evaluado distintas trayectorias con el sistema teleoperado. 
El artículo se divide del siguiente modo. En la sección 2, se presentan las distintas partes del sistema teleoperado. En la sección 3, se describe la integración de los distintos dispositivos. La sección 4 detalla el controlador propuesto. En la sección 5 se muestran los resultados obtenidos. Por último, en la sección 6 se presentan las conclusiones.

\section{DISPOSITIVOS DEL SISTEMA DE TELEOPERACIÓN}

A continuación, se describen los dispositivos que conforman el sistema de teleoperación propuesto.

\subsection{DISPOSITIVO HÁPTICO}

El dispositivo háptico utilizado para esta investigación es el modelo Omni Bundle desarrollado por la empresa Geomatic Touch. Posee seis grados de libertad, de los cuales, los tres primeros otorgan la ubicación y conjuntamente son actuados para dar la sensación háptica de fuerzas y los tres siguientes utilizados para la orientación, sin actuadores. Además, cuenta con dos botones físicos, capaces de ofrecer tres funciones, con pulsaciones independientes y mediante la combinación de ambos.

\subsubsection{Características del dispositivo Omni Bundle}

En la Figura 1 se muestra el diagrama cinemático del dispositivo háptico junto con sus grados de libertad.

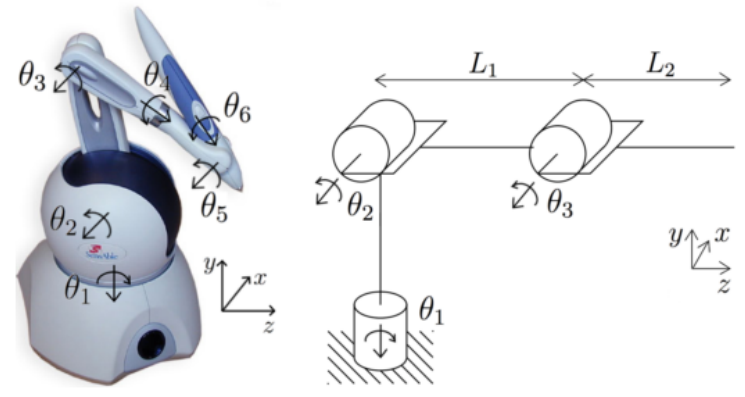

Figura 1. Diagrama cinemático para el Omni Bundle

De las especificaciones, las más destacables para este proyecto son: la fuerza que es capaz de ejercer, de $3.3 \mathrm{~N}$; la resolución en ubicación de aproximadamente $0.055 \mathrm{~mm}$; y el espacio de trabajo que, para mayor comprensión, se presenta en la Figura 2.
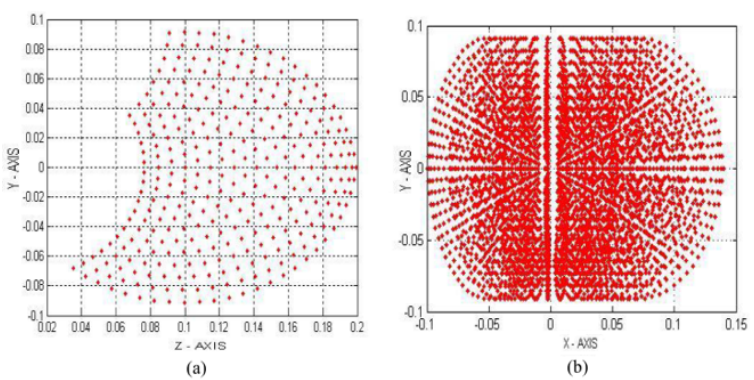

Figura 2. Espacio de trabajo del Omni Bundle. (a) Plano Y - Z, (b) Plano X - Y.

\subsubsection{Cinemática del dispositivo Omni Bundle}

Si bien ya existen estudios en los que se detallan las ecuaciones para describir el punto final del Omni Bundle [7], [2], se han hecho simplificando el modelo a tres grados de libertad o por el método geométrico. Por tanto, en este trabajo se propone caracterizar todo el dispositivo mediante el método de DenavitHartenberg, lo cual es conveniente para simular y visualizar todo el mecanismo, y no únicamente su punto final o efector. En la Figura 3 se exponen las consideraciones tomadas para la parametrización. Cabe remarcar que se ha añadido una distancia $l l$ desde el origen absoluto hasta el origen real del aparato, en caso de que sea necesario desplazar la altura de trabajo; además se ha cambiado el marco de referencia para coincidir con el sistema de referencia usado por el brazo MICO2. Igualmente se incluyen las medidas utilizadas y el rango de giro de cada articulación en la Tabla 1.

Tabla 1: Valores utilizados para la parametrización.

\begin{tabular}{|c|c|c|c|}
\hline Eslabón & $\begin{array}{c}\text { Distancia } \\
(\mathbf{m})\end{array}$ & Articulación & $\begin{array}{c}\text { Rango de } \\
\text { movimiento }\end{array}$ \\
\hline 11 & 0 & $\mathbf{q 1}$ & $\sim-55$ a 55 \\
\hline 12 & 0.15 & $\mathbf{q} 2$ & $\sim 0$ a 100 \\
\hline 13 & 0.133 & $\mathbf{q 3}$ & $\sim 110$ \\
\hline 14 & 0.073 & $\mathbf{q 4}$ & $\sim-145$ a 145 \\
\hline 15 & 0.06 & $\mathbf{q 5}$ & -80 a 60 \\
\hline 16 & 0 & $\mathbf{q 6}$ & -150 a 150 \\
\hline
\end{tabular}




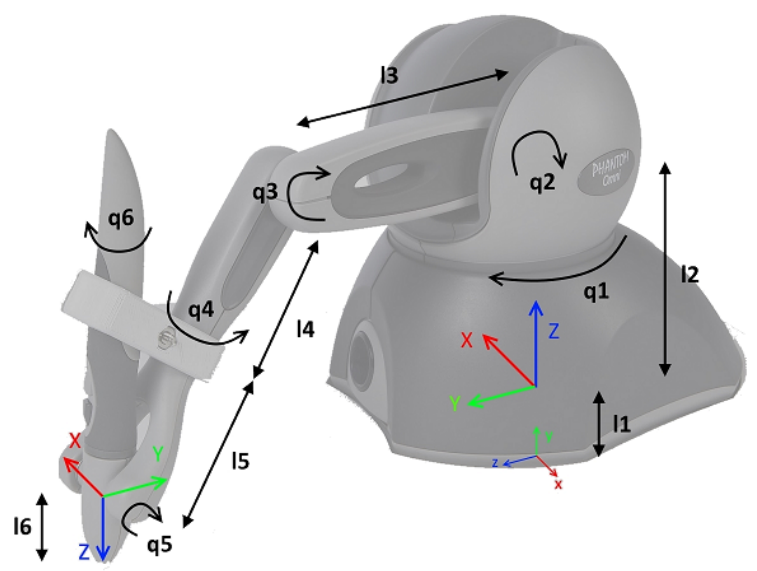

Figura 3. Esquema utilizado para la parametrización.

En función de lo anteriormente expuesto, los parámetros de Denavit-Hartenberg son los mostrados en la Tabla 2.

Tabla 2: Parámetros de Denavit-Hartenberg para el Omni Bundle.

\begin{tabular}{|c|c|c|c|c|}
\hline & Theta $(\boldsymbol{\theta )}$ & $\mathbf{d}$ & $\mathbf{a}$ & Alpha $(\boldsymbol{\alpha})$ \\
\hline $\mathbf{1}$ & 180 & 11 & 0 & 0 \\
\hline $\mathbf{2}$ & $-\mathrm{q} 1+90^{\circ}$ & 12 & 0 & $-90^{\circ}$ \\
\hline $\mathbf{3}$ & $\mathrm{q} 2+180^{\circ}$ & 0 & 13 & 0 \\
\hline $\mathbf{4}$ & $\mathrm{q} 3-\mathrm{q} 2$ & 0 & 0 & $90^{\circ}$ \\
\hline $\mathbf{5}$ & $-\mathrm{q} 4$ & $14+15$ & 0 & $-90^{\circ}$ \\
\hline $\mathbf{6}$ & $\mathrm{q} 5-90^{\circ}$ & 0 & 0 & $90^{\circ}$ \\
\hline
\end{tabular}

El sistema se comprobó mediante un GUI en Matlab que permite introducir las coordenadas articulares y observar la posición del dispositivo háptico en el espacio Cartesiano (ver Figura 4).

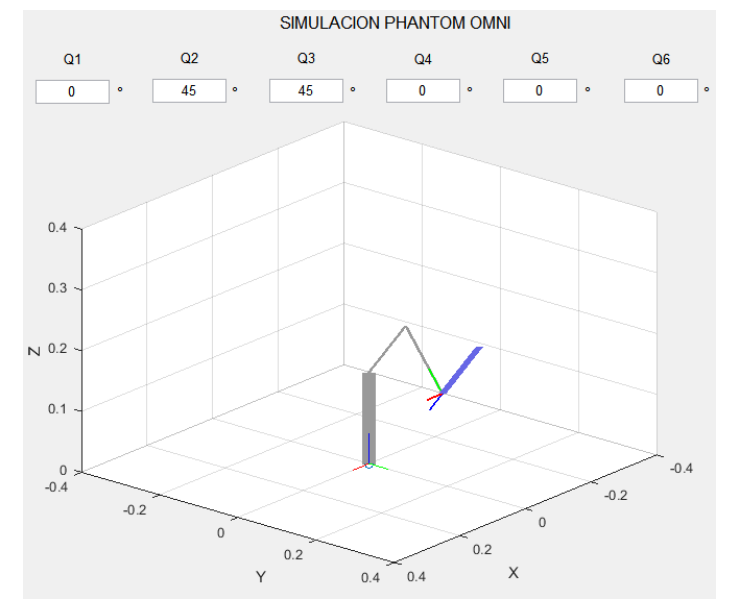

Figura 4. Interfaz gráfica de evaluación cinemática del dispositivo Omni Bundle.

Mediante comparación entre el diagrama mostrado por MATLAB y el dispositivo háptico real con los mismos ángulos, se corroboró que los parámetros de Denavit-Hartenberg son correctos.

\subsection{BRAZO ROBÓTICO}

El manipulador robótico empleado para este proyecto es la versión MICO2 de la empresa Kinova, junto con una pinza de tres dedos ( EError! No se encuentra el origen de la referencia. 5). Se trata de un brazo colaborativo, principalmente usado para brindar asistencia en entornos cotidianos a personas con algún impedimento físico o motriz.

\subsubsection{Características del brazo Kinova MICO2}

Como se dijo en el apartado anterior, el marco de referencia del brazo robótico es diferente al usado por el dispositivo háptico, en la Figura 5 se muestran los ejes que emplea el MICO2, y ya que será el mecanismo que se va a teleoperar, se adaptó el Omni Bundle para coincidir con éste. Además, se puede observar la notación para la orientación, siendo el ángulo $\theta_{x}$ el que lo orienta arriba/abajo, el ángulo $\theta_{y}$ alrededor de los dedos, y finalmente $\theta_{z}$ que es el giro circular de la muñeca.

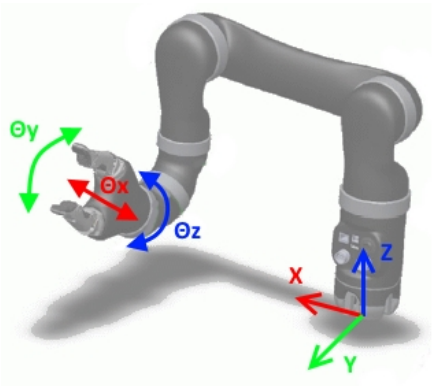

Figura 5. Ejes de referencia para el brazo robótico MICO2.

De las características principales del brazo robot, las más importantes para este proyecto son: alcance máximo de $70 \mathrm{~cm}$, peso de carga de aproximadamente $1 \mathrm{Kg}$, y el sistema de control que ofrece, Cartesiano o articular.

\section{INTEGRACIÓN DE LOS DISPOSITIVOS}

Una vez expuestas las características principales de cada dispositivo a utilizar, así como sus ejes de referencia, parametrización y métodos de control, se dispone a integrar ambos artefactos en el mismo espacio de trabajo. Si bien se permite el control articular del brazo robótico, y se pueden obtener los ángulos de cada articulación del mecanismo háptico, un control de este tipo no sería adecuado debido a que ambos dispositivos presentan distintos modelos cinemáticos, a pesar de que presenten igual número de 
grados de libertad. Debido a esto, se ha decidido usar el espacio Cartesiano XYZ para unificar los espacios de trabajo.

Mediante el modelado por Denavit-Hartenberg para el Omni Bundle, se hicieron coincidir los ejes de referencia, en consecuencia, ya se está trabajando sobre el mismo espacio de trabajo, con la diferencia de que el área útil del dispositivo háptico es menor a la del MICO2, lo que conlleva que deba existir un escalado en cada eje. Adicionalmente, hay que tener en cuenta que el brazo cuenta con una zona de protección alrededor del origen, un cilindro de aproximadamente $15 \mathrm{~cm}$ de radio. Estos conceptos se ilustran en la Figura 6. Finalmente, se hace notar que el espacio operativo del brazo se encuentra en el eje Y negativo, ya que hacia el eje Y positivo se encuentran los puertos de conexión y, por tanto, los cables.

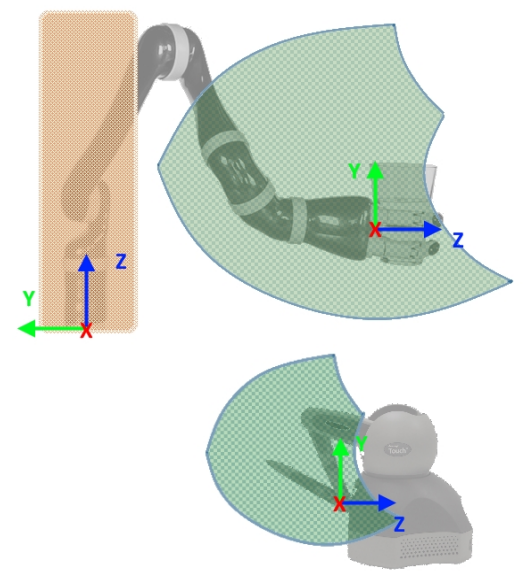

Figura 6. Espacio de trabajo Omni Bundle-MICO2

En virtud de lo expuesto anteriormente, la formulación matemática necesaria para ajustar la posición del brazo robótico respecto al dispositivo háptico se reduce a utilizar la ecuación de la recta, como se presenta a continuación.

$$
\begin{aligned}
& X_{M}=b_{x}+m_{x} * X_{P} \\
& Y_{M}=b_{y}+m_{Y} *\left(Y_{P}-0.27\right) \\
& Z_{M}=b_{z}+m_{Z} * Z_{P}
\end{aligned}
$$

donde el subíndice $M$ corresponde al $M I C O 2$ y el subíndice $P$ corresponde al Omni Bundle.

En la ecuación de la componente $\mathrm{Y}$ hay un ligero cambio debido a que se está trabajando en sentido negativo, como se explicó antes. El valor de 0.27 corresponde a los $270 \mathrm{~mm}$ que es el máximo alcanzable por el Omni Bundle en el eje Y.
El control sobre el manipulador, además de posición, incluye orientación, mediante los ángulos $\theta_{x}, \theta_{y}, \theta_{z}$; cuyo formato se encuentra en ángulos de Euler XYZ, los mismos que se pueden obtener de la submatriz de rotación de la matriz de transformación homogénea, $R$, tal como se indica a continuación.

$$
R=\left[\begin{array}{lll}
R_{11} & R_{12} & R_{13} \\
R_{21} & R_{22} & R_{23} \\
R_{31} & R_{32} & R_{33}
\end{array}\right]
$$

con:

- $\quad R_{11}=\mathrm{c}\left(\theta_{y}\right) \mathrm{c}\left(\theta_{z}\right)$

- $R_{12}=-\mathrm{c}\left(\theta_{y}\right) \mathrm{s}\left(\theta_{z}\right)$

- $R_{13}=\mathrm{s}\left(\theta_{y}\right)$

- $R_{21}=\mathrm{c}\left(\theta_{x}\right) \mathrm{s}\left(\theta_{z}\right)+\mathrm{c}\left(\theta_{z}\right) \mathrm{s}\left(\theta_{x}\right) \mathrm{s}\left(\theta_{y}\right)$

- $R_{22}=\mathrm{c}\left(\theta_{x}\right) \mathrm{c}\left(\theta_{z}\right)-\mathrm{s}\left(\theta_{x}\right) \mathrm{s}\left(\theta_{y}\right) \mathrm{s}\left(\theta_{z}\right)$

- $R_{23}=-\mathrm{c}\left(\theta_{y}\right) \mathrm{s}\left(\theta_{x}\right)$

- $R_{31}=\mathrm{s}\left(\theta_{x}\right) \mathrm{s}\left(\theta_{z}\right)-\mathrm{c}\left(\theta_{x}\right) \mathrm{c}\left(\theta_{z}\right) \mathrm{s}\left(\theta_{y}\right)$

- $R_{32}=\mathrm{c}\left(\theta_{z}\right) \mathrm{s}\left(\theta_{x}\right)+\mathrm{c}\left(\theta_{x}\right) \mathrm{s}\left(\theta_{y}\right) \mathrm{s}\left(\theta_{z}\right)$

- $R_{33}=\mathrm{c}\left(\theta_{x}\right) \mathrm{c}\left(\theta_{y}\right)$

donde $\mathrm{c}$ representa a la función coseno y s representa la función seno.

De forma que se pueden hallar los ángulos como sigue:

$$
\begin{aligned}
& \theta_{x}=\operatorname{atan} 2\left(\frac{-R_{23}}{R_{33}}\right) \\
& \theta_{y}=\operatorname{atan} 2\left(\frac{-R_{13}}{\sqrt{\left(R_{11}\right)^{2}+\left(R_{12}\right)^{2}}}\right) \\
& \theta_{z}=\operatorname{atan} 2\left(\frac{-R_{12}}{R_{11}}\right)
\end{aligned}
$$

donde atan2 representa la función inversa de la tangente en cuatro cuadrantes.

Nótese que existe una singularidad, denominada gimbal lock cuando el valor absoluto del elemento $R_{13}$ es igual a uno, ya que eso significa que $\sin \left(\theta_{y}\right)$ es la unidad, por consiguiente, $\cos \left(\theta_{y}\right)$ es nulo, lo que obliga a los elementos $R_{11}, R_{12}, R_{23}$ y $R_{33}$ a valer cero, $\mathrm{y}$ esto causa indeterminación en las ecuaciones para $\theta_{x}$ y $\theta_{z}$ antes descritas.

Para esta situación en particular, habrá que determinar los ángulos de la siguiente forma. Considerando que la matriz homogénea de rotación representa una terna ortonormal, así pues, cada elemento constituye entonces el coseno del ángulo entre los respectivos ejes que representan, esto significa que si el elemento $R_{13}$ tiene como magnitud la unidad, el ángulo entre el eje $\mathrm{X}$ y el eje $\mathrm{Z}$ de ambos sistemas de referencia es nulo, es decir, están alineados; y el ángulo en $\theta_{x}$ será 
el formado por los ejes $\mathrm{Y}$, esto es, el elemento $R_{22}$, deduciendo entonces que:

$$
\begin{aligned}
& \theta_{x}=\operatorname{acos}\left(R_{22}\right) \\
& \theta_{z}=0
\end{aligned}
$$

Donde el signo de $\theta_{x}$ estará determinado por el signo del elemento $R_{32}$, y $\theta_{y}$ se obtiene con la misma ecuación mostrada en (7).

Antes de implementar cualquier proyecto, y de ser posible, es conveniente simularlo antes, para detectar cualquier fallo u omisión que se haya cometido en el desarrollo del mismo. En este caso se ha hecho uso del programa en MATLAB elaborado en la sección anterior para el Omni Bundle modificado para que tenga comunicación con el software V-REP mediante las API remotas que ofrece el mismo, y además realice el escalado necesario para el espacio de trabajo del brazo MICO2. En la Figura 7 se muestran los cambios realizados, mostrando el valor de posición y los ángulos para cada dispositivo, varias opciones de vista, y un botón para iniciar la comunicación con V-REP.

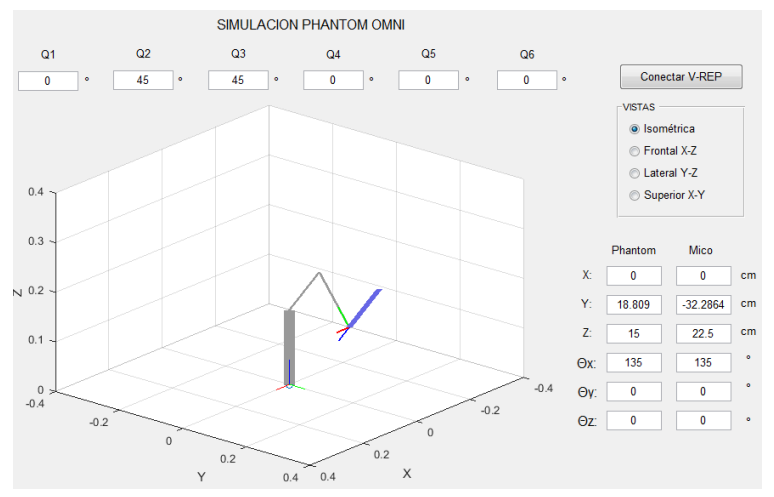

Figura 7. Interfaz gráfica en $M A T L A B$ para conectar con $V$-REP

\section{ESQUEMA DE CONTROL}

\subsection{FILTRADO DE LA SEÑAL DEL DISPOSITIVO HÁPTICO}

$\mathrm{Al}$ obtener la lectura de los ángulos del Omni Bundle y realizar las transformaciones necesarias para obtener la posición y orientación final del extremo del Kinova $\mathrm{MICO}$, resulta imprescindible implementar un filtro paso-bajo ya que, al manipular el dispositivo maestro, existen ligeras perturbaciones propias de un manejo manual, como vibraciones o movimientos involuntarios del operador. También es importante resaltar que, tal como se comentó en la sección 3, existe un factor de escalado para el espacio de trabajo del manipulador, de forma que estos movimientos indeseados se amplificarán en el efector final, debido a lo cual se propone aplicar un filtro para eliminar estos desplazamientos de alta frecuencia, aumentando así la precisión en la trayectoria.

La solución planteada ha sido implementar un filtro con respuesta impulsional infinita (IIR), debido a que proporciona una pendiente de corte más pronunciada y con menor retardo que un filtro de respuesta finita (FIR) [4]. Teniendo en cuenta que el ruido a eliminar no es muy prominente, se opta por un filtro de orden uno. El ruido se aprecia en las frecuencias mayores de $5 \mathrm{~Hz}$. Tras determinar la respuesta del filtro en frecuencia y fase por medio del diagrama de Bode, se obtiene la siguiente expresión para el filtrado de la señal leída a través del Omni Bundle.

$$
x=0.3 \cdot x_{\text {Anterior }}+0.7 \cdot x_{\text {Actual }}
$$

Se comprueba la respuesta efectuando una trayectoria al azar con el filtro implementado, obteniendo los resultados mostrados en la Figura 8, en los cuales se observa la atenuación de frecuencias mayores a cinco Herzios y, por tanto, se determina que el comportamiento es el deseado.
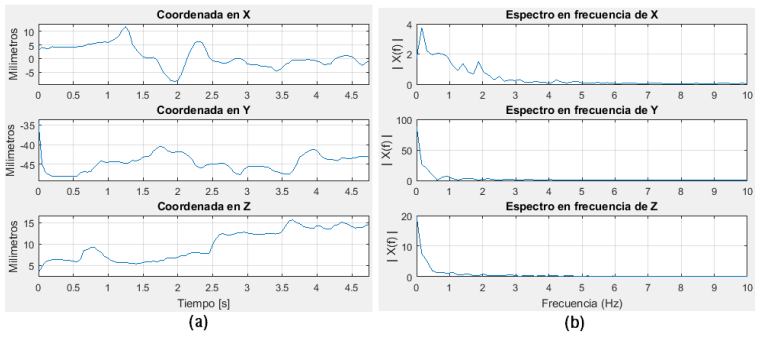

Figura 8. Trayectoria medida en el Omni Bundle con filtro. (a) Coordenadas en el tiempo, (b) Espectro frecuencial de las coordenadas

Utilizando el mismo procedimiento antes expuesto, se ha diseñado también un filtro de similares características para la realimentación de fuerza hacia el dispositivo háptico, debido a que el brazo robótico genera perturbaciones considerables mientras se desplaza.

Para controlar la posición del extremo del $\mathrm{MICO}$, se empleará el control cinemático Cartesiano de velocidad proporcionado por la API de programación del Kinova. Este modo de control permite enviar las velocidades deseadas al extremo del robot, tanto en traslación como en orientación, en un bucle de control con un tiempo de bucle muy pequeño. De esta forma, se obtiene una respuesta del manipulador con un retardo mínimo respecto al movimiento producido en el dispositivo háptico. Este retardo vendrá determinado por el esquema de comunicación utilizado, pero no por el esquema de control que se utiliza. Tras intentar realizar el control de cada coordenada Cartesiana mediante un controlador proporcional, se observó un error en régimen 
estacionario obtenido entre la posición demandada y la posición leída en el robot manipulador.

\subsection{CONTROLADOR PI}

La solución para eliminar el error en régimen permanente es bastante práctica, incrementar la velocidad de movimiento si a medida que el tiempo avanza, no se ha logrado llegar a la posición pretendida, o dicho matemáticamente, agregar una etapa integral al controlador. Con lo cual, la expresión requerida para implementar dicho controlador es la que se muestra en la ecuación (12).

$$
v=K_{p} \cdot e+K_{i} \cdot \sum_{n=0}^{\infty} e
$$

donde:

- $\quad v$ es el vector de velocidades a aplicar, de tamaño $6 \times 1$.

- $K_{p}$ es una matriz diagonal positiva de ganancias proporcionales, de tamaño $6 \times 6$.

- $e$ es el vector con las diferencias entre posición deseada y actual, de tamaño $6 \times 1$.

- $K_{i}$ es una matriz diagonal positiva de ganancias integrales, de tamaño $6 \times 6$.

Es pertinente aclarar que la formulación matemática correcta para la acción integral incluye una dependencia respecto al tiempo de muestreo, y dado que en esta aplicación se trata de una teleoperación, lo que significa retardos no homogéneos en el tiempo, se ha decidido no incluir esta variable, puesto que no es indispensable para su funcionamiento.

Para comprobar el correcto funcionamiento del controlador PI propuesto, se establecen los siguientes valores para las ganancias $K_{p}$ y $K_{i}$ :

$$
\begin{gathered}
K_{p}=\operatorname{diag}(2.1,2.1,2.1,3.5,3.5,3.5) \\
K_{i}=0.001 \cdot \mathbf{I}
\end{gathered}
$$

Como se puede apreciar en la Figura 9, el controlador funciona correctamente con el controlador PI propuesto. El seguimiento de la trayectoria es bastante cercano al deseado, y para aplicaciones en entornos cotidianos, como es el caso de este estudio, este control satisface los requerimientos. En la gráfica mencionada, se ilustran las trayectorias tanto para traslación como para orientación. Es destacable el hecho de que la orientación requiere un tiempo ligeramente mayor para alcanzar la consigna, mediante varias pruebas se ha llegado a la conclusión de que es una característica propia del controlador interno del MICO2. Otro rasgo considerable es el desfase que se puede apreciar entre la trayectoria consigna y la real, la cual se debe al retardo en la comunicación y al propio desfase introducido por el filtro que se comentó en la sección 4.1.

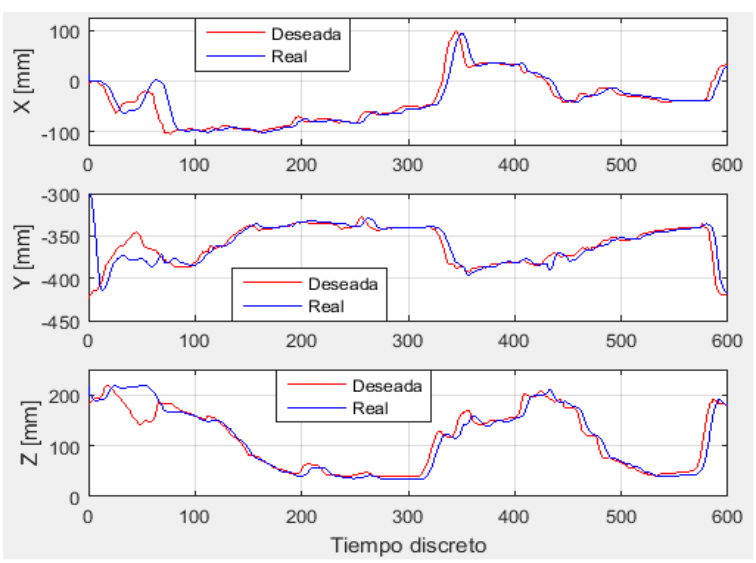

(a)

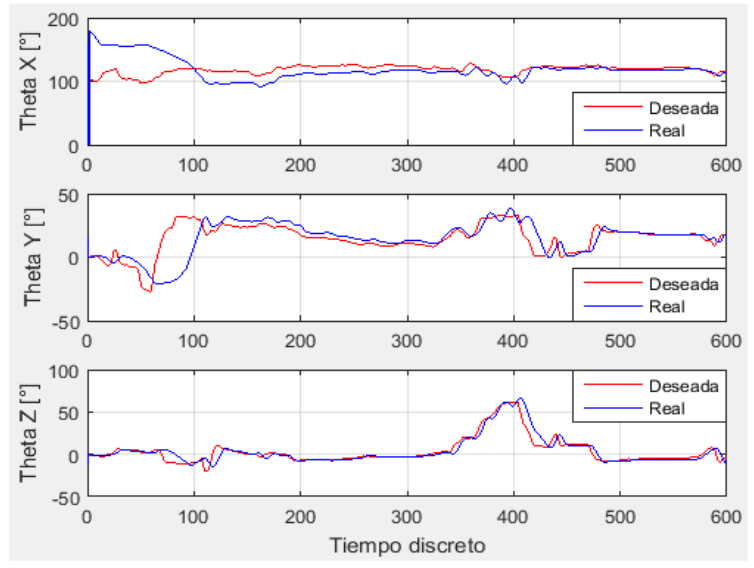

(b)

Figura 9. Control PI aplicado al brazo robótico. (a) Traslación, (b) Orientación

\section{RESULTADOS}

La Figura 10 muestra un experimento en el que se realiza básicamente un posicionamiento del robot manipulador en función del posicionamiento del Omni Bundle. En este primer experimento se trata de mostrar el funcionamiento del sistema de teleoperación abarcando todo el espacio de trabajo de ambos dispositivos, tanto en posición como en orientación.

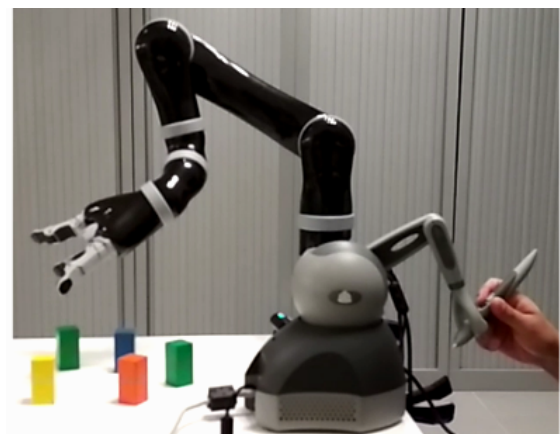


Figura 10. Sistema de teleoperación propuesto

El experimento funciona correctamente, proporcionando una muy buena sensación al usuario, tanto en la velocidad de respuesta como en la precisión. La Figura 11 muestra el recorrido realizado por la muñeca del Kinova MICO2 en el espacio Cartesiano. Se observa que el error se encuentra en el orden de los milímetros, lo cual es suficiente para aplicaciones cotidianas, como es el objetivo de esta investigación.
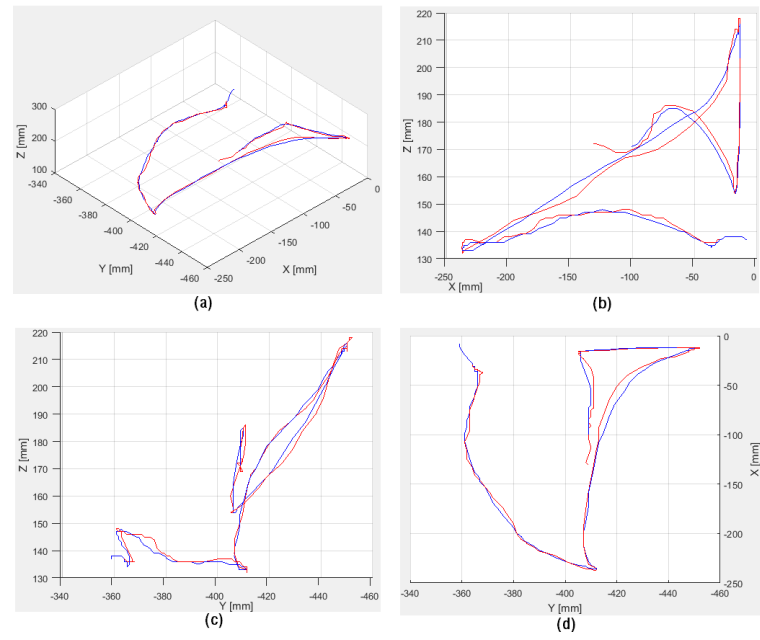

Figura 11. Trayectoria en espacio Cartesiano, en rojo la posición deseada y en azul la real. (a) Vista isométrica, (b) Plano XZ, (c) Plano YZ y (d) Plano $\mathrm{XY}$

En cuanto a la traslación, se puede acotar que el error es menor al centímetro, incluso si se tiene en cuenta que el cálculo del error está afectado por el desfase que existe entre ambas señales, ya que se puede valorar la similitud entre ambas si estuviesen en fase. Por otro lado, en orientación existe un error cercano a los diez grados sexagesimales. Aquí es pertinente acotar que al brazo robótico le resulta más difícil adquirir una nueva orientación manteniendo la posición fija. Por consiguiente, tarda más en llegar al valor consigna. Se ha preferido dejarlo con este comportamiento, ya que, al aumentar las ganancias de control para intentar reducirlo, se obtuvo el efecto contrario, llegando a la oscilación. La Figura 12 representa la evolución en cada uno de los ejes, tanto en traslación como en orientación. Se indica, además, el error promedio.
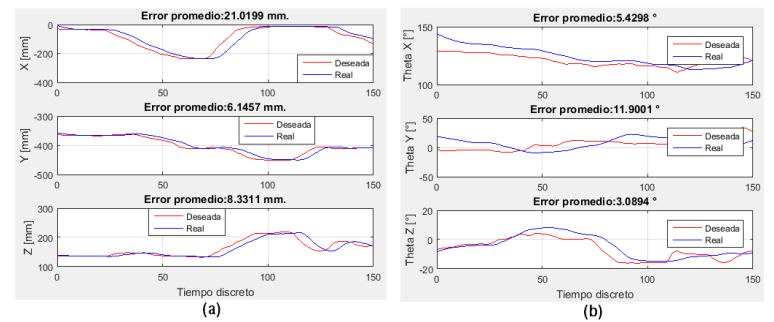

Figura 12. Trayectoria respecto al tiempo. (a) Traslación y (b) Orientación

Finalmente, se muestra un experimento donde se desea medir las fuerzas producidas en el robot de cara a comprobar el buen funcionamiento de la realimentación de fuerzas en el dispositivo háptico. Las fuerzas de perturbación se exponen en la Figura 13. Cabe recordar que el brazo Kinova MICO2 es capaz de soportar magnitudes de fuerza mayores de las que el dispositivo háptico puede generar, como en este caso particular, en el que la fuerza se encuentra alrededor de 25 N. por eje. El Omni Bundle sólo es capaz de producir $3.3 \mathrm{~N}$., motivo por el cual existe un factor de escalado que, para este experimento, fue de 5. Por tanto, se aplicó la máxima realimentación con estas perturbaciones.
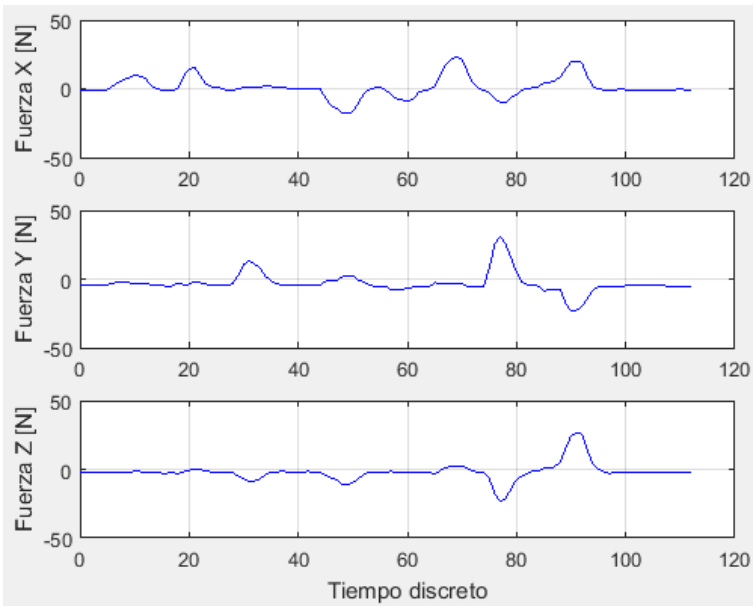

Figura 13. Componentes de fuerza medidas en el efector del brazo robótico

\section{CONCLUSIONES}

Los sistemas teleoperados surgieron como una necesidad de manipular objetos peligrosos sin exponer a las personas a dichos riesgos. A pesar de ello, la tecnología ha ido explorando nuevas aplicaciones para estos sistemas y, como es el caso de este estudio, en aplicaciones de uso común donde la robótica está ganando terreno día tras día. Con los resultados alcanzados, se presenta este método como válido para la teleoperación de un brazo robótico, dado que ha demostrado cumplir con especificaciones suficientes para ser manipulado y operado dentro de un régimen doméstico o comercial.

Mediante la experimentación y el uso de este sistema, se concluye que el manejo por parte de un dispositivo háptico maestro presenta mayor operatividad que otras interfaces de control como pueden ser: joystick o paneles de control ya sean físicos o virtuales; puesto que el manejo de una herramienta en el espacio 
Cartesiano resulta más intuitivo para un ser humano, a diferencia de objetos estáticos como botones, controles deslizantes o palancas, que no replican de manera exacta los movimientos que el efector del robot describirá. Así como también, por tener una referencia de la fuerza soportada por el manipulador, que permite percibir golpes, choques con objetos, o incluso el peso del objeto a operar.

Actualmente se está trabajando en el modelo dinámico del robot con el objetivo de diseñar un controlador dinámico articular que mejore todavía más la precisión y velocidad de reacción del sistema.

También se está desarrollando toda una batería de pruebas que permitirán establecer el nivel de mejora proporcionada a los usuarios a partir de la realimentación de fuerzas en el dispositivo háptico.

\section{Agradecimientos}

Este trabajo ha sido financiado por el Ministerio de Economía, Industria y Competitividad de España a través del proyecto DPI2015-68087-R, así como por los Fondos FEDER.

\section{English summary}

\section{TELEOPERATION OF KINOVA MICO2 ROBOT ARM USING AN OMNI BUNDLE DEVICE}

\begin{abstract}
In this work, a remote control of an assistive robot Kinova MICO2 using a haptic Omni Bundle device is presented. This device provides force feedback to increase the operator sensitivity. The most effective kinematic control method has been studied in accordance with the system requirements (position or velocity control) and evaluated with a graphical user interface in Visual Studio for monitoring and configuration. UDP has been established as communication protocol, as it is the best option in terms of speed and robot's reach. The results show that a velocity PI controller (proportional integral) is the most robust and allows performing teleoperation tasks with a very small error.
\end{abstract}

Keywords: teleoperation, haptic devices, assistive robots.

\section{Referencias}

[1] Fong, T., Thorpe, C., (2001) Vehicle teleoperation interfaces, Autonomous Robots, vol. 11, no. 1, pp. 9-18.

[2] Jarillo-Silva, A., Domínguez-Ramírez, O.A., Parra-Vega, V, Ordaz-Oliver, (2009) J.P, PHANToM OMNI haptic device: Kinematic and manipulability," Electronics, Robotics and Automotive Mechanics Conference (CERMA), pp. 193-198.

[3] Martin, H.L., Hamel, W.R., (1984) Joining teleoperation with robotics for advanced manipulation in hostile environments, Robots Conference.

[4] Mitra, S.K., (2001) Digital Signal Processing: A Computer-based Approach (2nd ed.), McGraw Hill, New York.

[5] Ni, D., Yew, A.W.W., Ong, S.K., Nee, A.Y.C. (2017) Haptic and visual augmented reality interface for programming welding robots," Advance Manufacturing, vol. 5, no. 3, pp. 191198.

[6] Nuño Ortega, N., Basañez, L., (2004) Teleoperación: técnicas, aplicaciones, entorno sensorial y teleoperación inteligente, Universidad Politécnica de Cataluña, pp. 1-43.

[7] Nygaard, A. (2018) High-Level Control System for Remote Controlled Surgical Robots," Norwegian University of Science and Technology.

[8] Olivieri, E., Barresi, G., Caldwell, D.G., Mattos, L.S., (2017) Haptic Feedback for Control and Active Constraints in Contactless Laser Surgery: Concept, Implementation and Evaluation, IEEE Transactions on Haptics, vol. 1412, no. c, pp. 114.

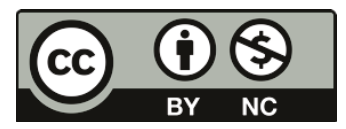

(C) 2018 by the authors. Submitted for possible open access publication under the terms and conditions of the Creative Commons Attribution CC-BY-NC 3.0 license (https://creativecommons.org/licenses/by-nc/3.0). 